\title{
Role of radiographs in shoulder pathology: a clinical review
}

This article was published in the following Dove Press journal:

Reports in Medical Imaging

12 July 2014

Number of times this article has been viewed

\author{
Oded Hershkovich ${ }^{1-3, *}$ \\ Shachar Shapira ${ }^{1-3, *}$ \\ Yaron Sela ${ }^{1,3}$ \\ Itamar B Botser ${ }^{1,3}$
}

'Department of Orthopedic Surgery, Chaim Sheba Medical Center, ${ }^{2}$ Medical Corps, Israeli Defense Forces, Tel Hashomer, ${ }^{3}$ Sackler Faculty of Medicine, Tel Aviv University, Tel Aviv, Israel

*SS and $\mathrm{OH}$ contributed equally to this work
Correspondence: Itamar B Botser Department of Orthopedic Surgery, Chaim Sheba Medical Center,

Tel Hashomer, 5262I, Israel

Tel +97235302623

Fax +972 35302523

Email itamar@botser.com
Abstract: Shoulder pain is a very common complaint, and affects as many as $20 \%$ of all people at some point during their lives. Despite the availability of more advanced imaging modalities, $\mathrm{X}$-ray remains the first imaging test to be performed in the investigation of any shoulder pain. However, with their increasing availability, ultrasound and magnetic resonance imaging have in recent years become first-line techniques for the diagnostic imaging of the shoulder. Moreover, ultrasound of the shoulder is increasingly performed in lieu of radiography. Nevertheless, many patients who visit a shoulder specialist for their pain are referred by a family physician or community orthopedist without an X-ray having ever been performed. Shoulder pain can be caused by many conditions, including rotator cuff tears, calcification within the tendons of the rotator cuff, stiff shoulder, subacromial impingement, space-occupying lesions, degenerative changes, and rheumatoid arthritis. This paper reviews various types of shoulder injury and the radiographic symptomatology of each, with the goal of encouraging the use of radiography by demonstrating the importance of this basic tool in the diagnostic process for these injuries.

Keywords: shoulder, pain, pathology, imaging, radiographs

\section{Introduction}

Shoulder pain is very common, with as many as $20 \%$ of the population suffering from shoulder pain during their lifetime. ${ }^{1}$ The prevalence of shoulder pain is second only to back pain among patients seeking treatment for musculoskeletal pain. ${ }^{2}$ Following a physical examination, radiography is still the first-line test in the examination of shoulder pain. ${ }^{3-8}$

With its increasing availability in recent years, ultrasound seems to have become competitive for the role as one of the first-line tests for the diagnosis of shoulder pain. Ultrasound of the shoulder is often performed to the exclusion of X-ray. Parallel to the growing use of ultrasound as a first-line test for the diagnosis of shoulder pain, magnetic resonance (MR) imaging along with MR tomography are becoming more common as a first-line diagnostic modality for shoulder pain. In line with this trend, many patients are referred to a shoulder specialist by a family physician or a community orthopedist without an X-ray taken.

As mentioned, X-ray of the shoulder should be the first imaging modality employed in the event of chronic or acute pain at or around the shoulder. ${ }^{3-8}$ Full radiological demonstration of the shoulder is recommended to include five views, ie, anteroposterior (AP), AP in internal rotation, AP in external rotation, an axillary view, and an outlet view. ${ }^{8}$ In our department, only three of these are routinely obtained, ie, AP, AP in internal rotation, and an axillary view. Patients in serious pain, for instance with a dislocation 
or fracture, may not be able to raise their arm for an axillary view or an internal rotation maneuver, in which case a transscapular (scapular Y) view is performed instead.

If the diagnosis remains unclear following radiography, additional imaging tests of the shoulder can be performed, including ultrasound, MR imaging, MR using contrast injected at the affected joint (MR arthrography), and computed tomography with or without contrast, in order to change the therapeutic process.

The purpose of this paper is to discuss the role of radiography in the diagnosis of shoulder injuries by reviewing the common causes of shoulder pain and providing a description of their characteristic radiographic findings.

\section{Shoulder impingement}

Impingement of the shoulder is a condition characterized by pressure on the supraspinatus tendon, the bursa of the subacromion, and the subdeltoid between the humeral head and the coracoacromial arch. Often, bony spurs can be seen in the acromion. Diagnosis is usually made by physical examination and based on disappearance of symptoms of impingement following injection of anesthetic into the shoulder. ${ }^{5,7}$ A higher prevalence of symptoms consistent with impingement is associated with the presence of certain anatomical variations, including os acromiale, a type 3 acromion according to the Bigliani classification, or an acromion with a back-sided sloping head.

Imaging of the origin of the shoulder (outlet) is used to determine the Bigliani classification of the acromion (type I, flat; type II, curved; type III, hooked), ${ }^{9}$ and to visualize an os acromiale. Studies conducted by Mayerhoefer et $\mathrm{al}^{10}$ have found that a radiographic outlet view of the shoulder is more reliable than an MR cross-section in determining the shape of the acromion, whereas ultrasound of the shoulder allows dynamic testing for impingement, as well as demonstration of thickening of the bursa. ${ }^{3}$

\section{Rotator cuff tendon injuries}

There are four rotator cuff muscles, ie, supraspinatus, infraspinatus, teres minor, and subscapularis. Most rotator cuff tendon tears occur in the supraspinatus and infraspinatus muscles, and may occur in an acute or chronic setting. Prevalence increases with age in the older population and can reach up to $50 \%$. The condition is asymptomatic in most patients. ${ }^{11-13}$ Physical examination is important for diagnosis, with the characteristic findings being pain on lifting the shoulder above 90 degrees (painful arc) and weakness of the rotator tendons..$^{2-4,8}$
Although it is not possible to visualize a torn tendon on X-ray, this modality is still recommended to begin with. An acute rupture usually appears normal on X-ray, ${ }^{5}$ but it is sometimes possible to detect a proximal migration of the head of the humerus into the subacromial space (in cases of massive long-standing rotator cuff tears). ${ }^{4}$ In addition, in patients with a traumatic rupture of the rotator cuff tendons due to high-energy injury (such as following dislocation), bone chips torn with the tendons may be visible on X-ray. In chronic ruptures of the rotator cuff tendons, in addition to the head shifting superiorly, changes may be observed to occur over time, eg, acromial bone spurs, enlarged tuberosity, and subacromial narrowing.

A study by Keener et $\mathrm{al}^{14}$ in patients with ruptures of tendons of the rotator cuff demonstrated greater upward shifting in shoulders of symptomatic patients versus asymptomatic patients. No significant difference in the size of the tears was noted between the two groups. Mayerhoefer et $\mathrm{al}^{15}$ found that the subacromial space, as measured by radiography and $\mathrm{MR}$, is in direct proportion to the condition of the shoulder as measured using the Constant score. No connection was found in the shape of the acromion based on Bigliani type.

Ultrasound may offer an easy and inexpensive option for identifying a rotator cuff tear, although it is important to remember that its diagnostic ability is operator-dependent., On the other hand, MR is a precise imaging technique that is considered less vulnerable to subjectivity and allows for visualization of the tear. Another advantage of MR is delineating the tear, as well as the condition of the muscles, which can undergo atrophy and changes in fat composition as a result of injury. According to Goutallier et al, fat-associated changes occurring in the muscles of the rotator cuff affect the surgical outcome. ${ }^{16}$

A large proportion of rotator cuff tendon tears worsen with time without surgical intervention. ${ }^{17}$ Progression of the rupture may lead to degenerative changes which develop as a result of the expanding tear of the tendon and from the upward shifting of the head. This injury, called rotator cuff arthropathy, is in contrast with osteoarthritis, in which tendons are preserved. Radiography can clearly visualize signs of this damage and aid in the planning of appropriate treatment. ${ }^{17,18}$ The radiographic signs in rotator cuff arthropathy are upward shift of the head, subacromial narrowing, bone spurs, joint space narrowing, greater tuberosity rounding, destruction of the inferior margin of the acromion, a socket-shaped lower part of the acromion (acetabularization), damage to the top of the glenoid, osteopenia of the acromion and the nearby humerus, and subdislocation of the glenohumeral joint (Figure 1). ${ }^{17}$ 


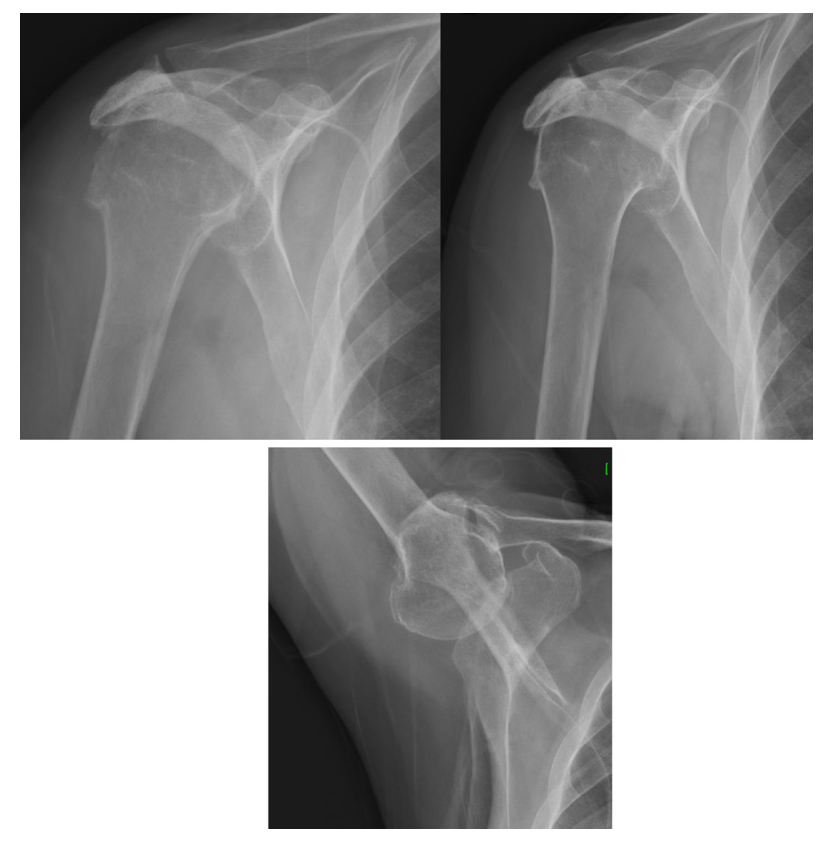

Figure I Classic finding in RC arthrophaty. Abbreviation: $\mathrm{RC}$, rotator cuff.

\section{Hydroxyapatite deposition disease}

Hydroxyapatite deposition disease, also known as calcific tendonitis, is a condition in which hydroxyapatite (calcium) deposits are formed within the tendons of the rotator cuff. The condition usually affects patients aged $30-50$ years, with a two-fold higher prevalence in women than in men. These deposits are bilateral in $10 \%-25 \%$ of patients. ${ }^{19}$

Calcific tendonitis has four stages: the precalcific stage (fibrocartilaginous metaplasia, fibrosis within the tendon); calcific/deposition stage (calcium precipitation and solidification); absorption stage (deposits are gradually absorbed); and postcalcific stage (healing of the tendon). ${ }^{19}$ Radiography allows for determination of which tendon contains the calcification and whether the condition is acute or chronic.

These calcifications appear most commonly in the tendon of supraspinatus and are visible over the greater tuberosity on images taken in the neutral position or internal rotation (Figure 2). However, calcifications occurring in the tendon of infraspinatus, teres minor, or subscapularis are better

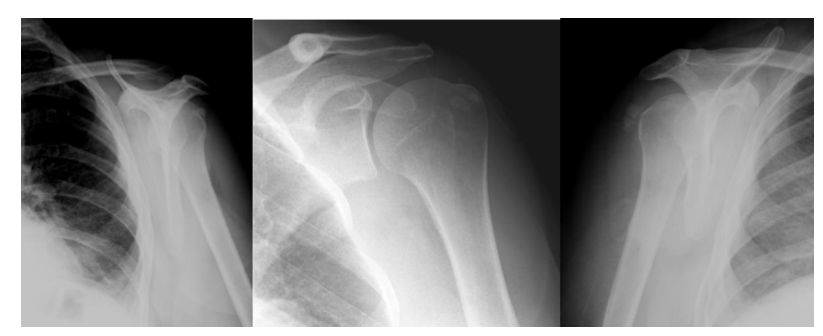

Figure 2 Hydroxyapatite deposition disease (calcific tendinitis). visualized in an axillary view (Figure 2). Calcification in the tendon of biceps brachii appears most commonly in the transition region between tendon and muscle or in the enthesis of the long tendon in the upper part of the glenoid. ${ }^{5}$

Chronic calcifications appear crowded with sharp boundaries. In contrast, new calcification appears less crowded, and is sometimes cloudy or with a cotton-ball appearance (Figure 2). ${ }^{5}$ Calcification in the deposition or absorption stages might be barely visible on X-ray. ${ }^{19}$ In a follow-up radiology study reported by Gartner, it was found that roughly $85 \%$ of loose (hazy) calcifications and $33 \%$ of crowded calcifications, considered classic markers for calcific tendinitis, disappeared on radiography within 3 years. ${ }^{20}$

Ultrasound is a very sensitive test for identifying calcification within the tendons, sometimes allowing for identification of calcification that cannot be viewed in other imaging modalities. Calcification can result in shadows in the imagery, which is easily mistaken for a tear in the tendons of the rotator cuff. ${ }^{19}$ Calcifications are usually not demonstrated in a clean manner on MR imaging, and since the result cannot aid in decision-making regarding treatment, the test is not recommended for this condition.

\section{Shoulder stiffness}

Significant shoulder pain combined with significant limitation in range of motion results in shoulder stiffness. There are three types of shoulder stiffness, ie, stiffness of unknown origin (idiopathic, also known as frozen shoulder or adhesive capsulitis), post-traumatic, and post-surgical.

\section{Adhesive capsulitis}

Adhesive capsulitis is associated with a gradual onset of pain and stiffness, and a history of diabetes or thyroid disease can support the diagnosis, ${ }^{4,21}$ while full range of motion of the shoulder excludes it. Shoulder stiffness is customarily classified into three stages: the first (freezing) stage usually varies in length from 2 to 9 months, pain is the most common initial symptom followed by gradual loss of range of motion and onset of stiffness, and the pain is usually more severe during the night and when the patient lies on the affected shoulder; the second (frozen) stage varies from 4 months to a year, during which the pain starts to withdraw despite worsening of stiffness and limitation in range of movement; and the third (thawing) stage ranges from 5 months to 3 years, during which the stiffness gradually disappears and the range of motion returns to normal or near normal levels.

Radiography will often appear normal, although a translucency of the head may sometimes be observed due to 
decreased use of the limb. ${ }^{3,4}$ Ultrasound of a frozen shoulder may demonstrate thickening of the joint capsule and increased fluid around the tendon of biceps brachii.

\section{Acromioclavicular joint pain}

Pain from the acromioclavicular joint is usually due to localized degenerative changes. A history of injury (for example, dislocation of the acromioclavicular joint) or weight lifting has been associated with this condition. Physical examination may reveal tenderness to palpation over the joint, a positive crossover sign, and pain with abduction above 150 degrees. ${ }^{4}$ Radiography can show narrowing of the joint space and bone spurs. Destruction of the distal part of the clavicle, or its elevation, also supports the diagnosis. A large number of individuals over the age of 40 years show degenerative changes of the acromioclavicular joint, but most are asymptomatic. ${ }^{22}$ The diagnosis is made by a combination of physical examination and radiographic findings. A bone scan as well as singlephoton emission computed tomography scan targeted to the acromioclavicular joint can also demonstrate this pathology. Ultrasound can be diagnostic for an acromioclavicular joint problem in a static or dynamic manner. ${ }^{2,23}$

\section{Glenohumeral instability}

Instability of the shoulder joint can be caused by damage to the joint capsule or labrum as a result of trauma or hyper-flexibility. Most affected patients are young (up to the age of 40 years), with a history of shoulder instability, dislocation, or subdislocation. In most cases, physical examination for shoulder instability will also be positive.

Radiography following shoulder dislocation may show a Hill-Sachs lesion, which is a compression fracture of the posterior part of the head, or a blurring of the anterior border of the glenoid, suggesting the presence of a bony Bankart lesion. Infrequently, fragments dislodged during the process of dislocation may be seen, and in older patients, avulsion fracture of the greater tuberosity by the rotator cuff tendons may accompany dislocation of the shoulder.

MR with contrast agent injected into the joint (MR arthrography) is considered by many to be the preferred diagnostic test in a patient with an unstable shoulder in order to demonstrate the non-bony structures of the shoulder, although this issue is still debatable. It will also show a Bankart lesion in a clear manner, even if does not involve a bony structure, as well as injuries to the edge of the joint or in the ligaments. Ultrasound does not allow for demonstration of the joint cartilage or injury at the edge of the joint. ${ }^{3}$

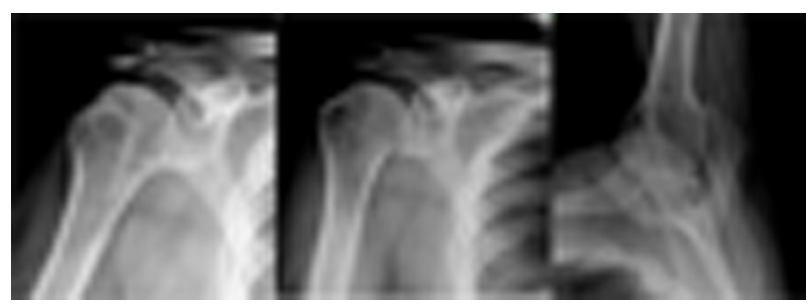

Figure 3 Osteoarthritis.

\section{Osteoarthritis}

The shoulder joint is not weight-bearing, and so osteoarthritis is less common in the shoulder than in other joints of the body. ${ }^{24}$ Most patients suffering from osteoarthritis have the condition are secondary to injury, systemic osteoarthritis, or acromegaly. The radiological signs are asymmetric stenosis of the joint space, inferior bone spurs at the anatomical neck of the glenoid, sclerosis, and subcartilaginous covering (Figure 3).

Unlike inflammatory conditions, bone density appears preserved. ${ }^{25,26}$ In advanced disease, a posterior subdislocation of the joint may occur. ${ }^{27}$ In most patients with primary osteoarthritis of the shoulder, the tendons of the rotator cuff are preserved. ${ }^{27}$ Radiography, together with physical examination, constitutes the best means of determining the diagnosis and the appropriate treatment. ${ }^{24}$ Ultrasound and MR may be performed prior to surgery, with the goal of demonstrating the integrity of the tendon of biceps brachii and the tendons of the rotator cuff. ${ }^{24}$ Computed tomography is recommended in order to demonstrate the deformity of the joint and to aid in planning of surgery. ${ }^{28}$

\section{Avascular necrosis}

Avascular necrosis of the humeral head has several causes, the most common being a post-traumatic state, steroid therapy, and sickle cell anemia. ${ }^{5}$ The X-ray findings depend on the stage of disease, and in most cases advance in the following sequence: formation of a transparent "crescent" or halfmoon shape (osteopenic bone), sclerosis, fragmentation, subchondral collapse, and, finally, degeneration and destruction of the

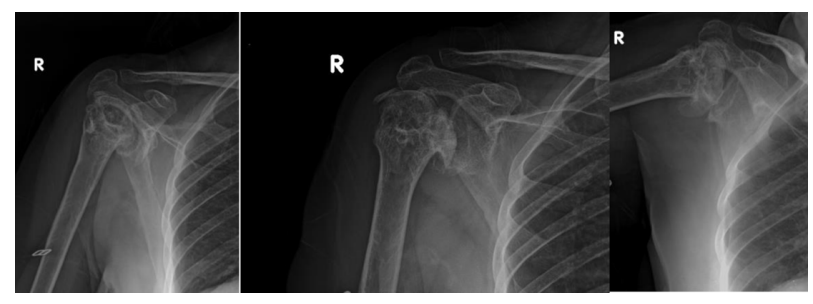

Figure 4 Avascular necrosis. Abbreviation: $\mathrm{R}$, right side. 


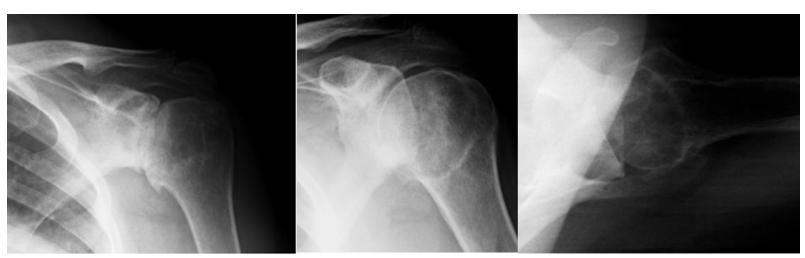

Figure 5 Rheumatoid arthritis.

joint $^{5}$ (Figure 4). The classic finding of avascular necrosis in its initial stages is a translucency in the shape of a half-moon (crescent sign), which is usually followed by the collapse of the subchondral bone of the head. MR and a bone scan can reveal avascular necrosis in its earlier stages. Ultrasound cannot assist in the diagnosis of avascular necrosis of the humeral head.

\section{Rheumatoid arthritis}

Radiological findings of rheumatoid arthritis in the shoulder include osteopenia, marginal erosions, and uniform damage to the cartilage (Figure 5); in contrast with osteoarthritis, bone spurs or sclerosis are not seen. ${ }^{5,25}$ The disease is often asymptomatic but symmetric, and presents as bilateral articular stenosis. Erosion often develops in the "bare area", ie, the part that is near the greater tubercle where the cartilage is thinner.

\section{Space-occupying lesions of the shoulder}

Space-occupying lesions of the shoulder typically present with pain and limitation in range of motion. ${ }^{29,30}$ In most cases, the
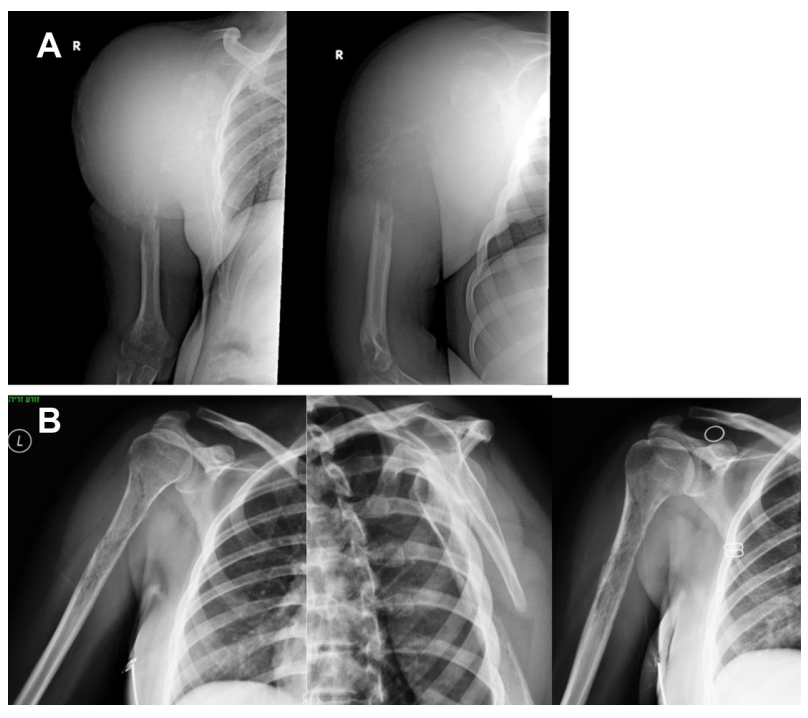

Figure 6 Space occupying lesions.

Note: the images $(\mathbf{A})$ and $(\mathbf{B})$ are examples of SOL.

Abbreviations: $\mathrm{R}$, right side; SOL, space-occupying lesions. lesion is seen on X-ray (Figure 6); however, when imagery is negative or not performed, the condition may be misdiagnosed as adhesive capsulitis. According to Robinson et al, ${ }^{30}$ local sensitivity that can be palpated on the bone is more typical for a space-occupying lesion than for a frozen shoulder. In children, a unicameral bone cyst can develop, which can lead to a pathological fracture. ${ }^{29}$ In older patients, malignant processes may spread to the nearby pouch. As mentioned, X-ray is the first-line diagnostic test for space-occupying processes. Bone scanning can help in diagnosis of space-occupying lesions of the bone; however, multiple myeloma without the presence of a fracture will not appear positive in the scan. Ultrasound also does not help with this diagnosis.

\section{Conclusion}

In our experience, all imaging investigations for shoulder pain should begin with an X-ray. Although not all causes of shoulder pain are clearly visible on radiography, this modality is still recommended as the starting point due to its wide availability and ready accessibility.

In patients with chronic shoulder pain, an X-ray supplies important basic information. It allows for: diagnosis of degenerative changes in the joint; visualization of an upward shift of the head of the humerus, which is a secondary sign of a large tear of the tendons of the rotator cuff; and also visualization of a downward shift of the head of the humerus in patients with bleeding inside the joint. In patients with chronic rotator cuff tendon tears, it allows visualization of changes in the head of the humerus, and sclerosis at the inferior portion of the acromion. X-ray also enables visualization of calcification inside the tendons, and bony processes which take up space.

In patients with trauma to the shoulder, the first test to be performed should be radiography. Dislocation of the shoulder or fracture of the proximal humerus is first diagnosed using $\mathrm{X}$-ray. X-ray is also recommended as the first step in patients with an acute rupture of a rotator cuff tendon; beyond the high position of the head of the humerus, it is sometimes possible to see a "pulling rupture" from the insertion area of the greater tubercle.

\section{Disclosure}

The authors report no conflicts of interest in this work.

\section{References}

1. Pope DP, Croft PR, Pritchard CM, Silman AJ. Prevalence of shoulder pain in the community: the influence of case definition. Ann Rheum Dis. 1997;56:308-312. 
2. Steinfeld R, Valente RM, Stuart MJ. A commonsense approach to shoulder problems. Mayo Clin Proc. 1999;74:785-794.

3. McNally EG, Rees JL. Imaging in shoulder disorders. Skeletal Radiol. 2007;36:1013-1016.

4. Burbank KM, Stevenson JH, Czarnecki GR, Dorfman J. Chronic shoulder pain: part I. Evaluation and diagnosis. Am Fam Physician. 2008;77:453-460.

5. Goud A, Segal D, Hedayati P, Pan JJ, Weissman BN. Radiographic evaluation of the shoulder. Eur J Radiol. 2008;68:2-15.

6. Shahabpour M, Kichouh M, Laridon E, Gielen JL, De Mey J. The effectiveness of diagnostic imaging methods for the assessment of soft tissue and articular disorders of the shoulder and elbow. Eur J Radiol. 2008;65:194-200.

7. Parker BJ, Zlatkin MB, Newman JS, Rathur SK. Imaging of shoulder injuries in sports medicine: current protocols and concepts. Clin Sports Med. 2008;27:579-606.

8. Green A. Chronic massive rotator cuff tears: evaluation and management. J Am Acad Ortho Surg. 2003;11:321-331.

9. Lu B, Morrison D, April, E. The morphology of the acromion and its relationship to rotator cuff tears. Orthop Trans. 1986;10:228.

10. Mayerhoefer ME, Breitenseher MJ, Roposch A, Treitl C, Wurnig C. Comparison of MRI and conventional radiography for assessment of acromial shape. AJR Am J Roentgenol. 2005;184:671-675.

11. Yamamoto A, Tagagishi K, Osawa T, et al. Prevalence and risk factors of a rotator cuff tear in the general population. J Shoulder Elbow Surg. 2010;19:116-120

12. Yamaguchi K, Ditsios K, Middleton WD, Hildebolt CF, Galatz LM, Teefey SA. The demographic and morphological features of rotator cuff disease. A comparison of asymptomatic and symptomatic shoulders. J Bone Joint Surg. 2006;88:1699-1704.

13. Reilly P, Macleod I, Macfarlane R, Windley J, Emery RJ. Dead men and radiologists don't lie: a review of cadaveric and radiological studies of rotator cuff tear prevalence. Ann R Coll Surg Engl. 2006;88: $116-121$.

14. Keener JD, Wei AS, Kim HM, Steger-May K, Yamaguchi K. Proximal humeral migration in shoulders with symptomatic and asymptomatic rotator cuff tears. J Bone Joint Surg. 2009;91:1405-1413.

15. Mayerhoefer ME, Breitenseher MJ, Wurnig C, Roposch A. Shoulder impingement: relationship of clinical symptoms and imaging criteria. Clin J Sport Med. 2009;19:83-89.

16. Goutallier D, Postel JM, Gleyze P, Leguilloux P, Van Driessche S. Influence of cuff muscle fatty degeneration on anatomic and functional outcomes after simple suture of full-thickness tears. J Shoulder Elbow Surg. 2003;12:550-554.
17. Ecklund KJ, Lee TQ, Tibone J, Gupta R. Rotator cuff tear arthropathy. J Am Acad Orthop Surg. 2007;15:340-349.

18. Feeley BT, Gallo RA, Craig EV. Cuff tear arthropathy: current trends in diagnosis and surgical management. J Shoulder Elbow Surg. 2009;18: 484-494.

19. Gosens T, Hofstee DJ. Calcifying tendinitis of the shoulder: advances in imaging and management. Curr Rheumatol Rep. 2009;11:129-134.

20. Gartner J. [Is tendinosis calcarea associated with HLA-A1?]. Z Orthop Ihre Grenzgeb. 1993;131:469. German.

21. Milgrom C, Novack V, Weil Y, Jaber S, Radeva-Petrova DR, Finestone A. Risk factors for idiopathic frozen shoulder. Isr Med Assoc J. 2008;10:361-364.

22. Pennington RG, Bottomley NJ, Neen D, Brownlow HC. Radiological features of osteoarthritis of the acromiclavicular joint and its association with clinical symptoms. J Orthop Surg (Hong Kong). 2008;16: 300-302.

23. Blankstein A, Ganel A, Givon U, et al. Ultrasonography as a diagnostic modality in acromioclavicular joint pathologies. Isr Med Assoc J. 2005;7:28-30.

24. Kircher J, Morhard M, Magosch P, Ebinger N, Lichtenberg S, Habermeyer P. How much are radiological parameters related to clinical symptoms and function in osteoarthritis of the shoulder? Int Orthop. 2010;346:767-681.

25. Wiater JM, Fabing MH. Shoulder arthroplasty: prosthetic options and indications. J Am Acad Orthop Surg. 2009;17:415-425.

26. Schwarzkopf R, Oron A, Loebenberg M. [Shoulder pain: assessment, diagnosis and treatment of common problems]. Harefuah. 2008;147: 71-76. Hebrew.

27. Iannotti JP, Norri TR. Influence of preoperative factors on outcome of shoulder arthroplasty for glenohumeral osteoarthritis. J Bone Joint Surg Am. 2003;85-A:251-258.

28. Nyffeler RW, Jost B, Pfirrmann CW, Gerber C. Measurement of glenoid version: conventional radiographs versus computed tomography scans. J Should Elbow Surg. 2003;12:493-496.

29. Cleeman E, Auerbach JD, Springfield DS. Tumors of the shoulder girdle: a review of 194 cases. J Should Elbow Surg. 2005;14:460-465.

30. Robinson D, Halperin N, Agar G, Alk D, Rami K. Shoulder girdle neoplasms mimicking frozen shoulder syndrome. J Should Elbow Surg. $2003 ; 12: 451-455$
Reports in Medical Imaging

\section{Publish your work in this journal}

Reports in Medical Imaging is an international, peer-reviewed, open access journal publishing original research, reports, reviews and commentaries on all areas of medical imaging. The manuscript management system is completely online and includes a very quick and fair peer-review system, which is all easy to use.
Dovepress

Visit http://www.dovepress.com/testimonials.php to read real quotes from published authors. 